\title{
One-step synthesis of size-tunable Ag nanoparticles incorporated in electrospun PVA/cyclodextrin nanofibers
}

\author{
Asli Celebioglu ${ }^{\mathrm{a}}$, Zeynep Aytac ${ }^{\mathrm{a}}$, Ozgun C.O. Umu ${ }^{\mathrm{a}}$, Aykutlu Dana ${ }^{\mathrm{a}}$, \\ Turgay Tekinay ${ }^{\mathrm{a}, \mathrm{b}, \mathrm{c}}$, Tamer Uyar ${ }^{\mathrm{a}, *}$ \\ a UNAM-Institute of Materials Science E Nanotechnology, Bilkent University, Ankara 06800, Turkey \\ ${ }^{\mathrm{b}}$ Life Sciences Application and Research Center, Gazi University, Ankara 06830, Turkey \\ c Gazi University, Polath Science and Literature Faculty, Ankara 06900, Turkey
}

\section{A R T I C L E I N F O}

\section{Article history:}

Received 10 May 2013

Received in revised form 18 July 2013

Accepted 27 August 2013

Available online 7 September 2013

\section{Keywords:}

Electrospinning

Nanofibers

Silver nanoparticles (Ag-NP)

Polyvinyl alcohol (PVA)

Cyclodextrin

\begin{abstract}
A B S T R A C T
One-step synthesis of size-tunable silver nanoparticles (Ag-NP) incorporated into electrospun nanofibers was achieved. Initially, in situ reduction of silver salt $\left(\mathrm{AgNO}_{3}\right)$ to $\mathrm{Ag}-\mathrm{NP}$ was carried out in aqueous solution of polyvinyl alcohol (PVA). Here, PVA was used as reducing agent and stabilizing polymer as well as electrospinning polymeric matrix for the fabrication of PVA/Ag-NP nanofibers. Afterwards, hydroxypropyl-beta-cyclodextrin (HP $\beta C D$ ) was used as an additional reducing and stabilizing agent in order to control size and uniform dispersion of Ag-NP. The size of Ag-NP was $\sim 8 \mathrm{~nm}$ and some Ag-NP aggregates were observed for PVA/Ag-NP nanofibers, conversely, the size of Ag-NP decreased from $\sim 8 \mathrm{~nm}$ down to $\sim 2 \mathrm{~nm}$ within the fiber matrix without aggregation were attained for PVA/HP $\beta C D$ nanofibers. The PVA/Ag-NP and PVA/HP $\beta C D / A g-N P$ nanofibers exhibited surface enhanced Raman scattering (SERS) effect. Moreover, antibacterial properties of PVA/Ag-NP and PVA/HP $\beta C D / A g-N P$ nanofibrous mats were tested against Gram-negative (Escherichia coli) and Gram-positive (Staphylococcus aureus) bacteria.
\end{abstract}

(c) 2013 Elsevier Ltd. All rights reserved.

\section{Introduction}

Electrospinning has become an attractive and a practical technique for the production of nanofibers and nanofibrous non-woven mats because of its versatility and a cost-effective setup (Ramakrishna, 2005; Wendorff, Agarwal, \& Greiner, 2012). Electrospun nanofibrous mats have very high specific surface area, nanoscale porous structures and unique chemical, physical, mechanical and surface properties (Greiner \& Wendorff, 2007; Ramakrishna, 2005; Ramakrishna et al., 2006; Wendorff et al., 2012). Electrospinning has an exceptional advantage over conventional fiber production techniques since nanofibers can be readily produced from a variety of polymers, polymer blends, sol-gels, suspensions, emulsions and composite structures. It has been shown that electrospun nanofibers and their nanofibrous mats can be particularly useful in filtration, tissue engineering, drug delivery, energy, sensors, electronics and environment applications due to their distinctive properties and specific functionalities (Greiner \& Wendorff, 2007; Guiping, Dawei, Yang, Xiaodan, \& Jun, 2012; Li \& Xia, 2004; Ramakrishna, 2005; Ramakrishna et al., 2006; Wendorff et al., 2012).

\footnotetext{
* Corresponding author. Tel.: +90 3122903571; fax: +90 3122664365

E-mail addresses: tamer@unam.bilkent.edu.tr, uyar@unam.bilkent.edu.tr, tameruyar@gmail.com (T. Uyar).
}

Electrospun nanofibers have design flexibility for particular functionalization which can be performed during the electrospinning process or by applying post-treatment methods. For instance, functional composite nanofibers can be produced by incorporation of metal nanoparticles (NP), such as silver or gold, into electrospun polymeric nanofibers (Hang, Tae, \& Park, 2010; Xiao, Xu, Ma, \& Fang, 2012; Zhu et al., 2012). However, the size and homogeneous distribution of metal NP through the nanofiber matrix should be taken into consideration to achieve high efficiency and effective performance from these nanofibrous composite materials. For example, silver nanoparticles (Ag-NP) have attracted considerable attention due to their unique optical, electronic, catalytic and antibacterial properties (Arvizo et al., 2012; Fouda, El-Aassar, \& Al-Deyab, 2013; Rycenga et al., 2011). Several studies aimed at incorporation of Ag-NP within electrospun nanofibers which can enable production of functional nanofibrous composites by combining the unique properties of nanofibers with that of Ag-NP (Mahanta \& Valiyaveettil, 2012; Nam et al., 2010; Shi et al., 2011; Xiao et al., 2012). Yet, the size control and uniform distribution of Ag-NP without aggregation in the polymeric nanofiber matrix is challenging, therefore, a number of different approaches were followed for obtaining electrospun polymer/Ag-NP composite nanofibers (Jin et al., 2007; Li et al., 2006; Patel, Li, Wang, Zhang, \& Wei 2007; Xiao et al., 2012; Xu et al., 2006). However, in these approaches silver salt precursor was directly added to the polymer solution and the synthesis of Ag-NP was carried out through 

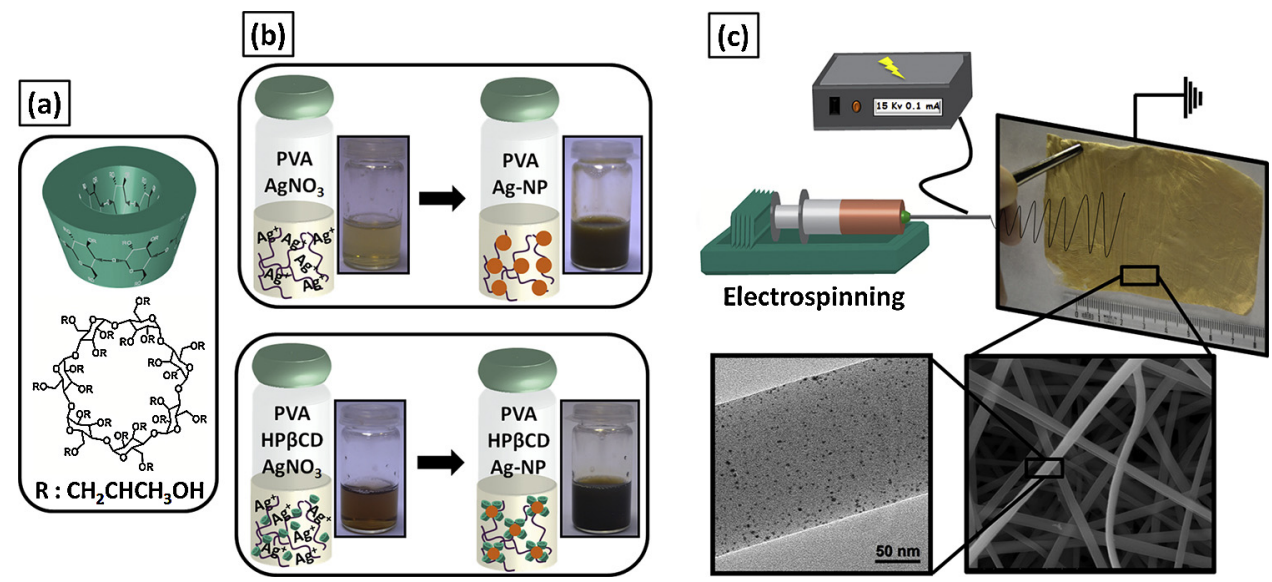

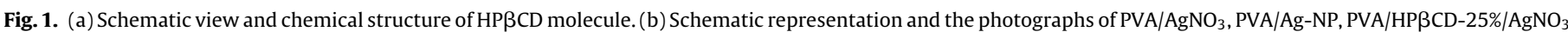

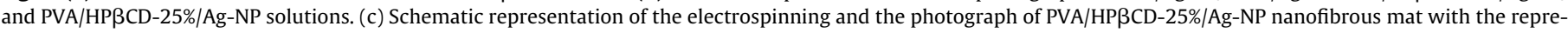
sentative SEM and TEM images.

thermal (Jin et al., 2007; Patel et al., 2007), chemical (Xiao et al., 2012; Xu et al., 2006) and photoreductive (Li et al., 2006) posttreatment of the electrospun nanofibers. So these above mentioned methods are often complex, time-consuming, and mostly require reducing and stabilizing chemicals which are sometimes highly toxic.

Therefore, simple and environmentally friendly approaches are essential for the practical applications of electrospun nanofibers incorporating Ag-NP. For example, one-step synthesis of Ag-NP incorporated in polyethylene oxide nanofibers (Saquing, Manasco, \& Khan, 2009) or polyvinyl alcohol nanofibers (Mahanta \& Valiyaveettil, 2012) was achieved where the polymer solution was used as both a reducing and protecting agent for Ag-NP and an electrospinning template. In other studies, polymer/Ag-NP composite nanofibers were produced in one-step by using the electrospinning solvent as a reducing agent and the electrospinning polymer matrix as a stabilizing/protective agent (Shi et al., 2011; Wang, Bai, Li, Zhang, \& Zhang, 2012).

The use of toxic reducing and stabilizing agents for the synthesis of Ag-NP were replaced with so-called "green" substances such as natural biomolecules including cellulose, chitosan, polyphenols, ascorbic acid and cyclodextrins (Cai, Kimura, \& Kuga, 2009; Laudenslager, Schiffman, \& Schauer, 2008; Ng, Yang, \& Fan, 2008; Ravi, Christena, SaiSubramanian, \& Anthony 2013; Wu et al., 2011). As well, "green" practices started to take their place in the electrospinning process by the use of natural and nonhazardous polymers (Mahanta \& Valiyaveettil, 2012). Very recently, polymer/cyclodextrin $/ \mathrm{AgNO}_{3}$ mixtures were electrospun into polymer/Ag-NP composite nanofibers where the cyclodextrin was used as a stabilizing and reducing agent for the formation of Ag-NP in the polymer matrix (Chae, Kim, Yang, \& Rhee, 2011; Wang, Bai, Li, \& Zhang, 2012). However, polymer used in these studies was dissolved in dimethylformamide (DMF) which is an undesirable solvent type from the point of biomedical applications. On the other hand, cyclodextrins (CD) are natural and non-toxic cyclic oligosaccharides which are produced by enzymatic degradation of starch. CD have a truncated cone-shaped molecular structure which enables them to form host-guest inclusion complexes with a variety of compounds via non-covalent interactions (Fig. 1a). Therefore, $C D$ are used in a number of industrial areas such as pharmaceuticals, food, chromatography, cosmetics and textiles (Chung, Guo, Priestley, \& Kwak, 2011; Harada, Kobayashi, Takashima, Hashidzume, \& Yamaguchi, 2010; Hedges, 1998; Szejtli, 1998). In addition to that, being a non-toxic and naturally occurring material, $C D$ are also very promising candidates for use as reducing and stabilizing agent for the formation of metal NP (Alvarez, Liu, Román, \& Kaifer, 2000; Huang, Meng, \& Qi, 2009; Kochkar, Aouine, Ghorbel, \& Berhault, 2011; Liu et al., 2012; Ng et al., 2008).

In this study, we report a one-step synthesis of Ag-NP incorporated into electrospun composite nanofibers. Initially, we used a biocompatible polymer, polyvinyl alcohol (PVA), as reducing agent to convert silver salt $\left(\mathrm{AgNO}_{3}\right)$ into $\mathrm{Ag}-\mathrm{NP}$ and then we obtained PVA/Ag-NP composite nanofibers via electrospinning. In addition to that, we also used hydroxypropyl-beta-cyclodextrin (HP $\beta C D$ ) as both reducing and stabilizing agent to control the size and uniform dispersion of Ag-NP within the electrospun nanofibers. The PVA/HP $\beta C D$ solution having different amount of HP $\beta C D$ were prepared to investigate the effect of HP $\beta C D$ amount on the size of Ag-NP and their dispersion within the nanofiber matrix. It was observed that the size of Ag-NP decreases significantly and homogeneous distribution of Ag-NP without aggregation were achieved in the electrospun PVA/HP $\beta C D$ nanofibers. The detailed morphological and structural characterizations of the samples were performed by scanning electron microscope (SEM), transmission electron microscope (TEM), X-ray diffractometer (XRD), UV-Vis-NIR spectroscopy, X-ray photoelectron spectroscopy (XPS) and Raman spectroscopy. Surface enhanced Raman scattering (SERS) properties of these electrospun nanofibers incorporating Ag-NP have been investigated. The antibacterial property of Ag-NP is well-known and widely studied (Guzman, Dille, \& Godet, 2012; Morones et al., 2005; Rai, Yadav, \& Gade, 2009). Hence, we have also performed antibacterial tests for PVA/Ag$\mathrm{NP}$ and PVA/HP $\beta \mathrm{CD} / \mathrm{Ag}-\mathrm{NP}$ composite nanofibrous mats against Gram-negative (Escherichia coli) and Gram-positive (Staphylococcus aureus) bacteria.

\section{Experimental}

\subsection{Materials}

The hydroxypropyl-beta-cyclodextrin (HP $\beta C D$, substitution: 0.6-0.9) was purchased from Wacker Chemie AG (Germany) commercially. The polyvinyl alcohol (PVA, Scientific Polymer, 88\% hydrolyzed, Mw 125,000), silver nitrate $\left(\mathrm{AgNO}_{3}\right.$, Sigma Aldrich, $\geq 99.5 \%$ ) and sodium hydroxide ( $\mathrm{NaOH}$, Fluka, $\geq 98 \%$, small beads) were purchased. The deionized water was used from the Millipore Milli-Q Ultrapure Water System. All the materials were used without any purification. 

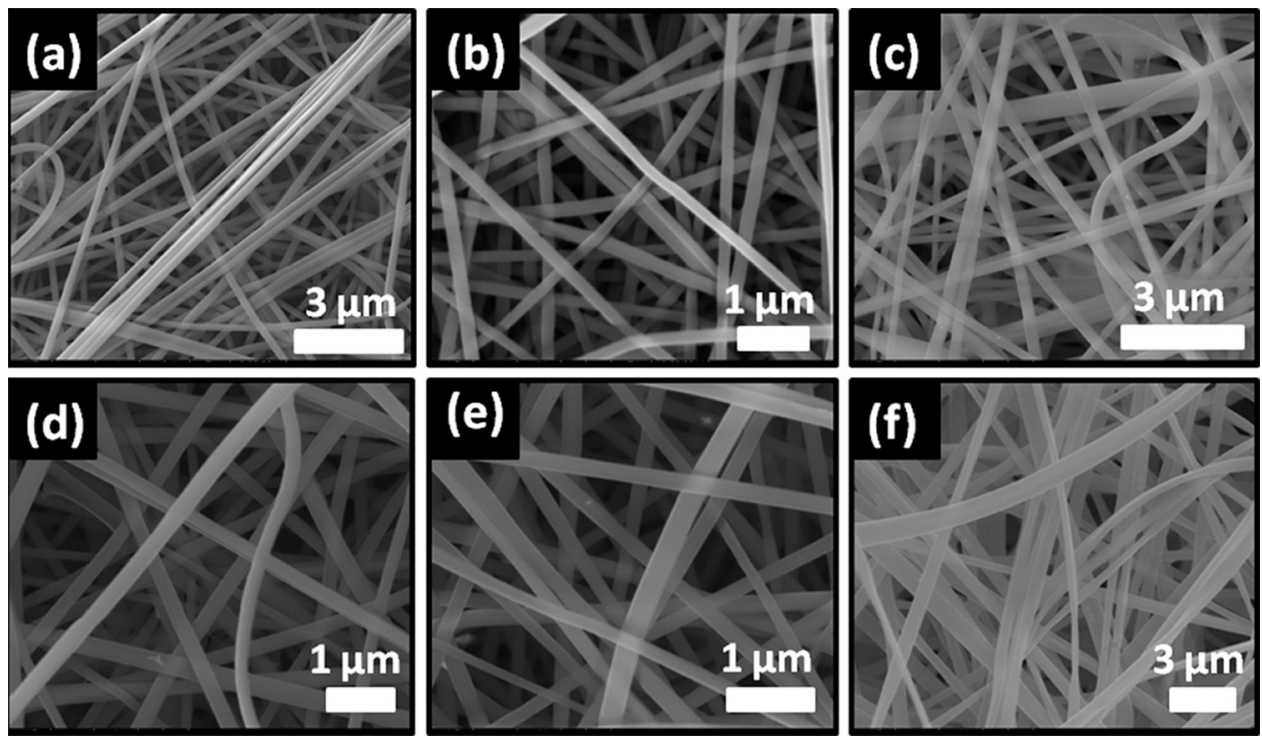

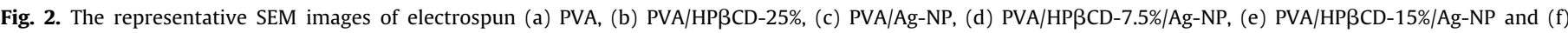
PVA/HP $\beta C D-25 \% / A g-N P$ nanofibers.

\subsection{Electrospinning}

First, the aqueous PVA solution was prepared by using 7.5\% ( $w / v$, with respect to solvent) concentration. On the other hand, HP $\beta C D$ was added to the aqueous PVA solution $(7.5 \%, w / v)$ at three different concentrations $(7.5 \%, 15 \%$ and $25 \%, \mathrm{w} / \mathrm{v}$, with respect to solvent). After obtaining a clear and homogeneous aqueous solutions of PVA and PVA/HPßCD, $\mathrm{AgNO}_{3}$ was added to each of these solutions and the concentration of $\mathrm{AgNO}_{3}$ was adjusted accordingly to have $1 \%(\mathrm{w} / \mathrm{w}$, with respect to total PVA or PVA/HP $\beta C D$ concentration) elemental $\mathrm{Ag}$ in the electrospun nanofibers. After the $\mathrm{AgNO}_{3}$ was dissolved completely, $\mathrm{pH}$ of the systems were adjusted to $\sim 8.5$ by adding $1 \mathrm{M} \mathrm{NaOH}$ to the solutions. The $\mathrm{pH}$ of PVA solution without HPßCD was also adjusted to the same $\mathrm{pH}$ level to keep the medium similar to each other. As the solutions were stirred overnight, the dark brown solutions were obtained indicating the formation of Ag-NP in the electrospinning solutions. For comparison, PVA (7.5\%, w/v) and PVA (7.5\%, w/v)/HP $\beta C D(25 \%, w / v)$ solutions without containing $\mathrm{AgNO}_{3}$ were also prepared for electrospinning. Each prepared solution was loaded into $3 \mathrm{~mL}$ syringes (metallic needle with 0.6 inner diameter) and positioned horizontally on the syringe pump (Model: SP 101IZ, WPI). The electrode of the high voltage power supply (Matsusada Precision, AU Series) was clamped to the metal needle tip of the syringe and the cylindrical aluminum collector was grounded. The electrospinning of the solutions was performed at the following parameters: applied voltage: $15 \mathrm{kV}$, tip-to-collector distance: $15 \mathrm{~cm}$ and the solution flow rate: $0.5 \mathrm{~mL} / \mathrm{h}$. Electrospun composite nanofibers were deposited on a grounded stationary cylindrical metal collector covered by a piece of aluminum foil. The electrospinning apparatus was enclosed in a Plexiglas box and the electrospinning was carried out at $25^{\circ} \mathrm{C}$ and $25 \%$ relative humidity.

\subsection{Measurements and characterization}

A rheometer (Anton Paar Physica CR 301) equipped with a cone/plate accessory (spindle type CP40-2) was used to measure the rheological behavior of the electrospinning solutions in the range of $0.1-100 \mathrm{~s}^{-1}$ shear rate. The conductivity of the solutions was measured with a Multiparameter InoLab $^{\circledR}$ Multi 720 (WTW) at room temperature. The morphological characterizations of nanofibers were carried out by using the scanning electron microscope (SEM) (Quanta 200 FEG, FEI). Samples were sputtered with $5 \mathrm{~nm} \mathrm{Au} / \mathrm{Pd}$ (PECS-682) and the average fiber diameter (AFD) was calculated from the SEM images by analyzing at least 100 fibers. Transmission electron microscope (TEM) (FEI-Tecnai G2F30) was used for the detection of Ag-NP in the nanofiber structure. For TEM imaging, HC200 grids were attached on the aluminum foil and the nanofiber samples were directly electrospun onto the grids. The average particle sizes of Ag-NP were determined from GATAN digital micrograph program. X-ray diffractometer (XRD) (X'Pert powder diffractometer, PANalytical) was used to determine the X-ray diffraction pattern of the nanofiber samples with $\mathrm{Cu} \mathrm{K} \alpha$ radiation in the range of $2 \theta=25-80^{\circ}$. The UV-Vis-NIR spectrophotometer (Varian Cary 5000, USA) was used in the wavelength range of 400-800 $\mathrm{nm}$ to observe characteristic absorption of Ag-NP. The UV-vis spectra were obtained by dissolving the nanofibers in water. The background was corrected with the aqueous solution of pure PVA and PVA/HP $\beta C D$ nanofibers without Ag-NP. The X-ray photoelectron spectra of the nanofibers were recorded by using $X$ ray photoelectron spectrometer (XPS) (Thermo Scientific). XPS was used by means of a flood gun charge neutralizer system equipped with a monochromated $\mathrm{Al} \mathrm{K \alpha} \mathrm{X}$-ray source $(h v=1486.6 \mathrm{eV})$. In order to obtained detailed information, the high resolution spectra were recorded for the spectral regions relating to silver at pass energy of $50 \mathrm{eV}$. Raman measurements are performed using a WITEC Alpha 300S system. A diode-pumped solid-state $532 \mathrm{~nm}$ wavelength laser is used for excitation in the Raman measurements. Laser power has been calibrated using a silicon photodiode at sample plane.

\subsection{Antibacterial test}

The antibacterial activities of the nanofibers were performed against Escherichia coli RSHM 888 (RSHM, National Type Culture Collection Laboratory, Ankara, Turkey) as a Gram-negative bacteria and Staphylococcus aureus RSHM 96090/07035 (ATCC 25923) as a Gram-positive bacteria. The nanofibrous mats were cut into circular discs having diameter of $0.8 \mathrm{~cm}$. The disk agar diffusion method was conducted. $150 \mu \mathrm{L}$ of the overnight grown cultures $\left(\sim 10^{1} \mathrm{cfu} / \mathrm{mL}\right.$ of E. coli and $\sim 10^{9} \mathrm{cfu} / \mathrm{mL}$ of $S$. aureus) were spreaded on Luria-Bertani (LB) agar. The nanofibrous mats were placed on top of the agar 
Table 1

The property of the electrospinning solutions and the average fiber diameter and the average particle size of Ag-NP present in the electrospun nanofibers.

\begin{tabular}{|c|c|c|c|c|}
\hline Sample & $\begin{array}{l}\text { Conductivity } \\
(\mu \mathrm{S} / \mathrm{cm})\end{array}$ & $\begin{array}{l}\text { Viscosity } \\
\text { (Pa s) }\end{array}$ & $\begin{array}{l}\text { Average fiber } \\
\text { diameter }(\mathrm{nm})\end{array}$ & $\begin{array}{l}\text { Average particle } \\
\text { size of Ag-NP }(\mathrm{nm})\end{array}$ \\
\hline PVA & 525 & 0.50 & $290 \pm 75$ & - \\
\hline PVA/HP $\beta C D-25 \%$ & 830 & 0.60 & $500 \pm 140$ & - \\
\hline PVA/Ag-NP & 1415 & 0.35 & $235 \pm 40$ & $8.0 \pm 0.5$ \\
\hline PVA/HPßCD-7.5\%/Ag-NP & 1630 & 0.60 & $360 \pm 70$ & $2.7 \pm 0.5$ \\
\hline PVA/HP $\beta C D-15 \% / A g-N P$ & 1625 & 0.63 & $400 \pm 70$ & $2.6 \pm 0.5$ \\
\hline PVA/HP $\beta C D-25 \% / A g-N P$ & 1635 & 0.70 & $485 \pm 100$ & $1.8 \pm 0.4$ \\
\hline
\end{tabular}

plate. The Petri dishes were incubated at $37^{\circ} \mathrm{C}$ for $24 \mathrm{~h}$. The tests were repeated three times for each of bacteria. The zones where the bacterial growth was not observed were recorded as inhibition zones and diameters were measured.

\section{Results and discussion}

\subsection{Electrospinning and morphological characterizations of PVA/Ag-NP and PVA/HP $\beta C D / A g-N P$ composite nanofibers}

Here, in situ reduction of silver nitrate $\left(\mathrm{AgNO}_{3}\right)$ into silver nanoparticle (Ag-NP) was achieved in polyvinyl alcohol (PVA) aqueous solution where PVA was acted as both reducing agent and stabilizing agent as well as electrospinning polymer matrix for the fabrication of PVA/Ag-NP composite nanofibers. PVA can reduce the $\mathrm{Ag}^{+}$ions into $\mathrm{Ag}-\mathrm{NP}$ due to the hydroxyl groups on the polymer backbone (Mahanta \& Valiyaveettil, 2012) and also stabilize the Ag-NP by keeping them from aggregation. Yet, in order to control the size and ensure uniform dispersion of Ag-NP in PVA solution, we also used hydroxypropyl-beta-cyclodextrin (HPßCD) as a supplementary reducing and stabilizing agent. The carbohydrates (glucose, starch, etc.) have high potential for the reduction of $\mathrm{AgNO}_{3}$ to the Ag-NP; however to be more efficient in the ambient conditions, $\mathrm{NaOH}$ could be added to the system to enhance and accelerate the reduction of $\mathrm{Ag}^{1+}$ to $\mathrm{Ag}^{0}$ by releasing the electrons from glucose molecules (Shervani, \& Yamamoto, 2011), otherwise, these agents represent limited reducing effect. So, we have prepared the alkaline conditions by using $\mathrm{NaOH}(\mathrm{pH} \sim 8.5)$ to benefit from the reducing potential of HP $\beta C D$ molecules that have very similar chemical structure with other types of carbohydrates. The concentration of PVA in aqueous solution was adjusted as 7.5\% $(w / v$, with respect to solvent) in order to obtain bead-free and uniform nanofibers by electrospinning. For PVA/HP $\beta C D$ blend solutions, the concentration of PVA was kept at 7.5\% (w/v) and three different concentrations of HP $\beta C D$ was used; $7.5 \%, 15 \%$ and $25 \%$ $(\mathrm{w} / \mathrm{v})$. Previously, we have shown that HPßCD can be electrospun
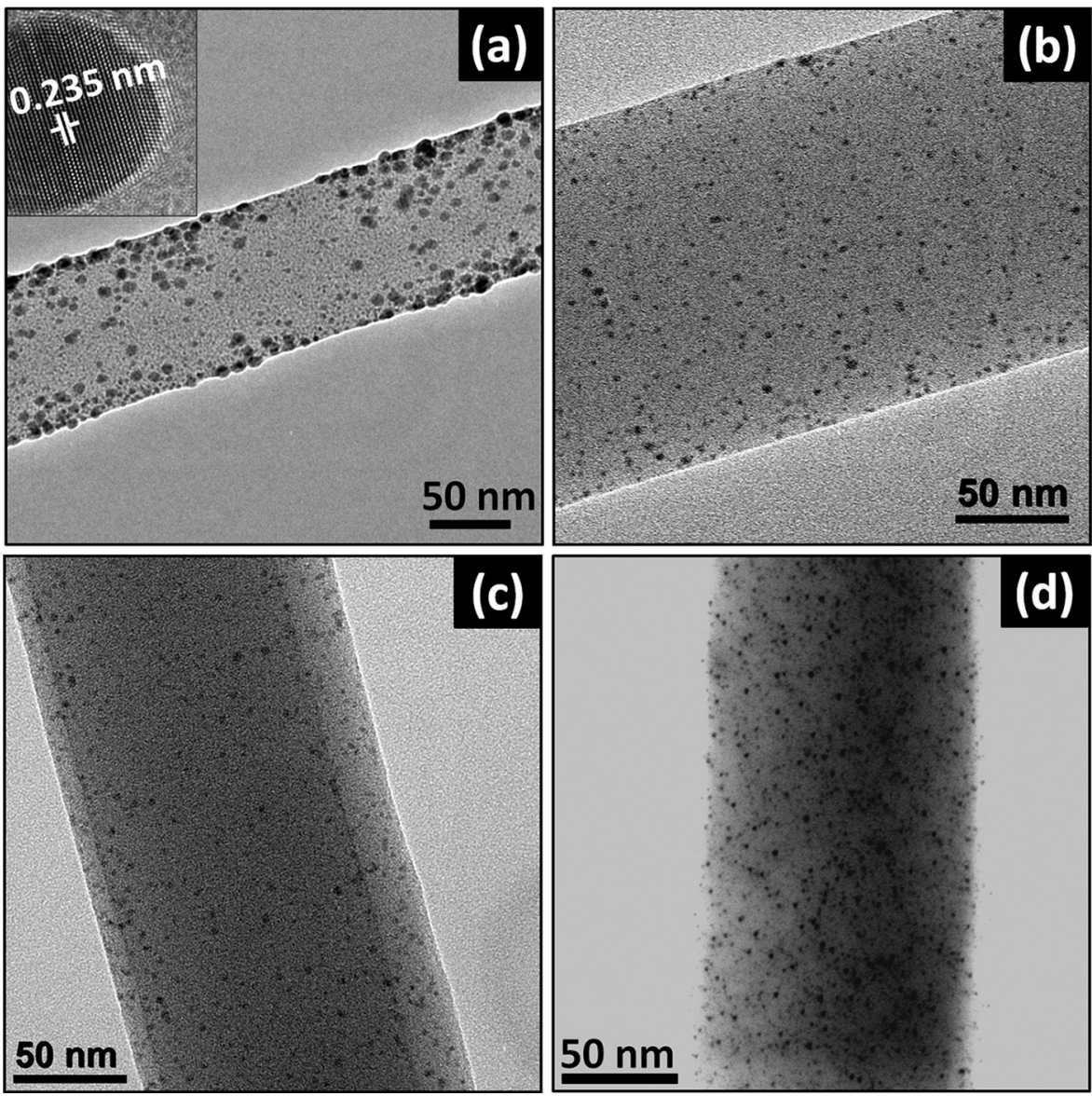

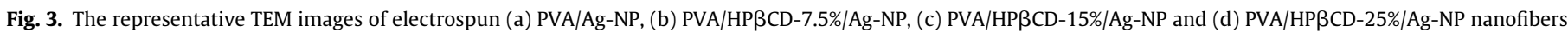
The HR-TEM image of a single Ag-NP indicating the $d$-spacing between $\mathrm{Ag}\left\{\begin{array}{lll}1 & 1 & 1\end{array}\right\}$ planes as inset figure. 
by itself without using polymeric matrix, but, much higher concentration (up to $160 \%(\mathrm{w} / \mathrm{v})$ ) was required for obtaining uniform nanofibers (Celebioglu \& Uyar, 2012). $\mathrm{AgNO}_{3}$ was added to homogeneous aqueous solutions of PVA and PVA/HP $\beta C D$ and the dark brown solutions were obtained after overnight mixing indicating the Ag-NP formation in the electrospinning solutions (Fig. 1b). In each electrospinning solution, the concentration of $\mathrm{AgNO}_{3}$ was adjusted accordingly to have $1 \%(\mathrm{w} / \mathrm{w}$, with respect to total PVA or PVA/HP $\beta C D$ concentration) Ag-NP in the electrospun nanofibers.

The electrospinning of PVA/HP $\beta C D / A g-N P$ nanofibers was illustrated in Fig. 1c. The electrospun PVA/HP $3 C D / A g-N P$ composite nanofibrous mats have the characteristic color of Ag-NP and these materials are flexible which can be easily handled as a free-standing mat (Fig. 1c). The representative SEM images of PVA, PVA/HP $\beta C D$, PVA/Ag-NP and PVA/HPßCD/Ag-NP nanofibers were shown at Fig. 2 and their average fiber diameters (AFD) were given in Table 1 . In all cases, bead-free and uniform nanofibers were obtained elucidating that the concentration and viscosity of the electrospinning solutions were at the optimal level. However, the AFD of the nanofibers were different from each other because of the differences in the viscosity and conductivity values of the solutions (Table 1). In electrospinning, typically high solution viscosity or low solution conductivity yield thicker fibers because of the less stretching of the electrospinning jet (Ramakrishna, 2005; Wendorff et al., 2012). On the contrary, electrospinning of solutions having lower viscosity or higher solution conductivity resulted in thinner fibers because of the more stretching of the electrospinning jet (Ramakrishna, 2005; Wendorff et al., 2012). The electrospun PVA nanofibers have AFD of $290 \pm 75 \mathrm{~nm}$ and when HPBCD was added to the PVA solution at the highest HP $\beta C D$ concentration (25\%, w/v), PVA/HP $\beta C D-25 \%$ nanofibers having AFD of $500 \pm 140 \mathrm{~nm}$ were obtained. Although the solution conductivity increased a bit, high solution viscosity of PVA/HP $\beta C D-25 \%$ yielded thicker fibers. In the case of electrospun PVA/Ag-NP nanofibers, the AFD decreased to $235 \pm 40 \mathrm{~nm}$ when compared to PVA nanofibers and this was owing to the presence of Ag-NP which contributed to higher solution conductivity (Saquing et al., 2009) and the viscosity of the solution was also lowered which resulted in more stretching of the electrospinning jet. The PVA/HP $\beta C D / A g-N P$ solutions have higher viscosity and conductivity values, in addition, the higher amount of HP $B C D$, the higher the viscosity of the solutions were obtained and the solution conductivity of the PVA/HP $\beta C D / A g-N P$ systems were very close to each other. Therefore, thicker fibers were expected to be produced as the amount of HP $\beta C D$ increased from $7.5 \%(w / v)$ through $25 \%(w / v)$. As expected, we observed that the AFD of PVA/HP $\beta C D-$ 7.5\%/Ag-NP, PVA/HPßCD-15\%/Ag-NP and PVA/HPßCD-25\%/Ag-NP nanofibers has AFD of $360 \pm 70 \mathrm{~nm}, 400 \pm 70 \mathrm{~nm}$ and $485 \pm 100 \mathrm{~nm}$, respectively.

The representative TEM images of PVA/Ag-NP and PVA/HP $\beta C D / A g-N P$ nanofibers were shown in Fig. 3 and the average particle size (APS) of Ag-NP present in the fiber matrix was given in Table 1. Ag-NP were seen as black spherical spots in TEM images of the fiber samples. For PVA/Ag-NP nanofiber sample, the APS of the Ag-NP was $8.0 \pm 0.5 \mathrm{~nm}$ but some Ag-NP aggregations were also observed for this sample (Fig. $3 a$ ). On the other hand, it was apparent that Ag-NP have homogenous distribution through the fiber matrix for PVA/HP $3 C D / A g-N P$ samples (Fig. 3b-d). Additionally, the size of Ag-NP decreases significantly in the PVA/HP $\beta C D / A g-N P$ nanofiber samples as the amount of HPBCD increases in the fiber matrix. The APS of the Ag-NP was $8.0 \pm 0.5 \mathrm{~nm}$ for $\mathrm{HP} \beta C D$ free PVA/Ag-NP nanofibers whereas the size of the Ag-NP decreased to $2.7 \pm 0.5 \mathrm{~nm}, 2.6 \pm 0.5 \mathrm{~nm}$ and $1.8 \pm 0.4 \mathrm{~nm}$ for PVA/HPßCD-7.5\%/Ag-NP, PVA/HP $\beta C D-15 \% / \mathrm{Ag}-\mathrm{NP}$ and PVA/HP $\beta C D-25 \% / A g-N P$ nanofiber samples, respectively.

Owing to hydroxyl groups, PVA polymer has the ability to reduce $\mathrm{Ag}$ salts into Ag-NP13, however, with the addition of HP $\beta C D$ in the
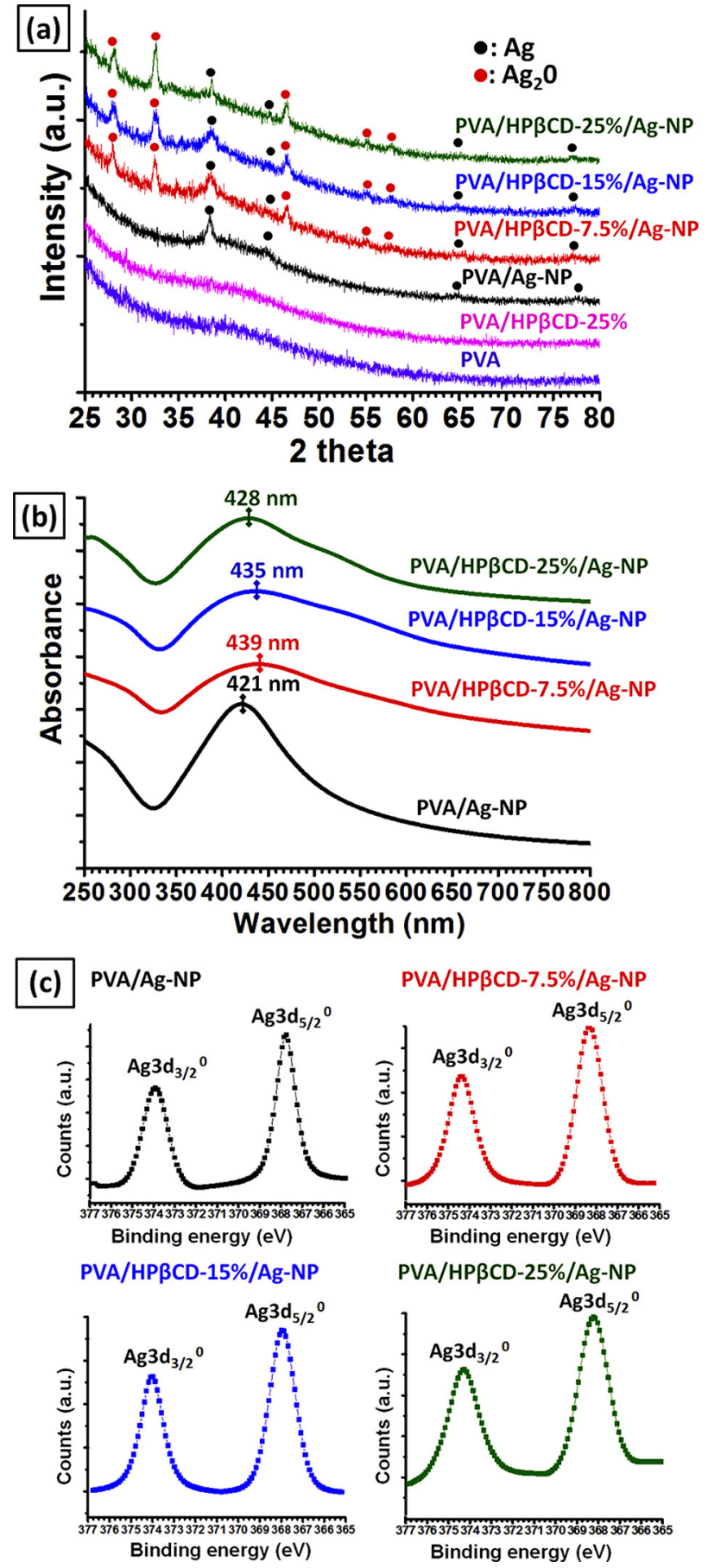

Fig. 4. (a) XRD spectra of PVA, PVA/HPßCD-25\%, PVA/Ag-NP, PVA/HPßCD-7.5\%/AgNP, PVA/HPßCD-15\%/Ag-NP and PVA/HP $3 C D-25 \% / A g-N P$ nanofibrous mats. (b) UV-vis spectra taken from the dissolved PVA/Ag-NP, PVA/HP $\beta C D-7.5 \% / A g-N P$, PVA/HP $\beta C D-15 \% / A g-N P$ and PVA/HP $\beta C D-25 \% / A g-N P$ nanofibers in water. (c) The high resolution XPS of electrospun PVA/Ag-NP, PVA/HPßCD-7.5\%/Ag-NP, PVA/HP $\beta$ CD-15\%/Ag-NP and PVA/HP $\beta C D-25 \% / A g-N P$ nanofibers.

PVA solution significant decrease was observed for the size of AgNP due to the highly efficient stabilizing and reducing properties of CD molecules (Kochkar et al., 2011; Ng et al., 2008). Furthermore, Ag-NP did not aggregate or coagulate locally due to the stabilizing effect of HP $\beta C D$ that leads to uniform distribution of Ag-NP within the fiber matrix. More importantly, the size Ag-NP can be controlled by varying the amount of HP $\beta C D$, that is, higher the amount of $\mathrm{HP} \beta C D$, smaller the size of Ag-NP. 

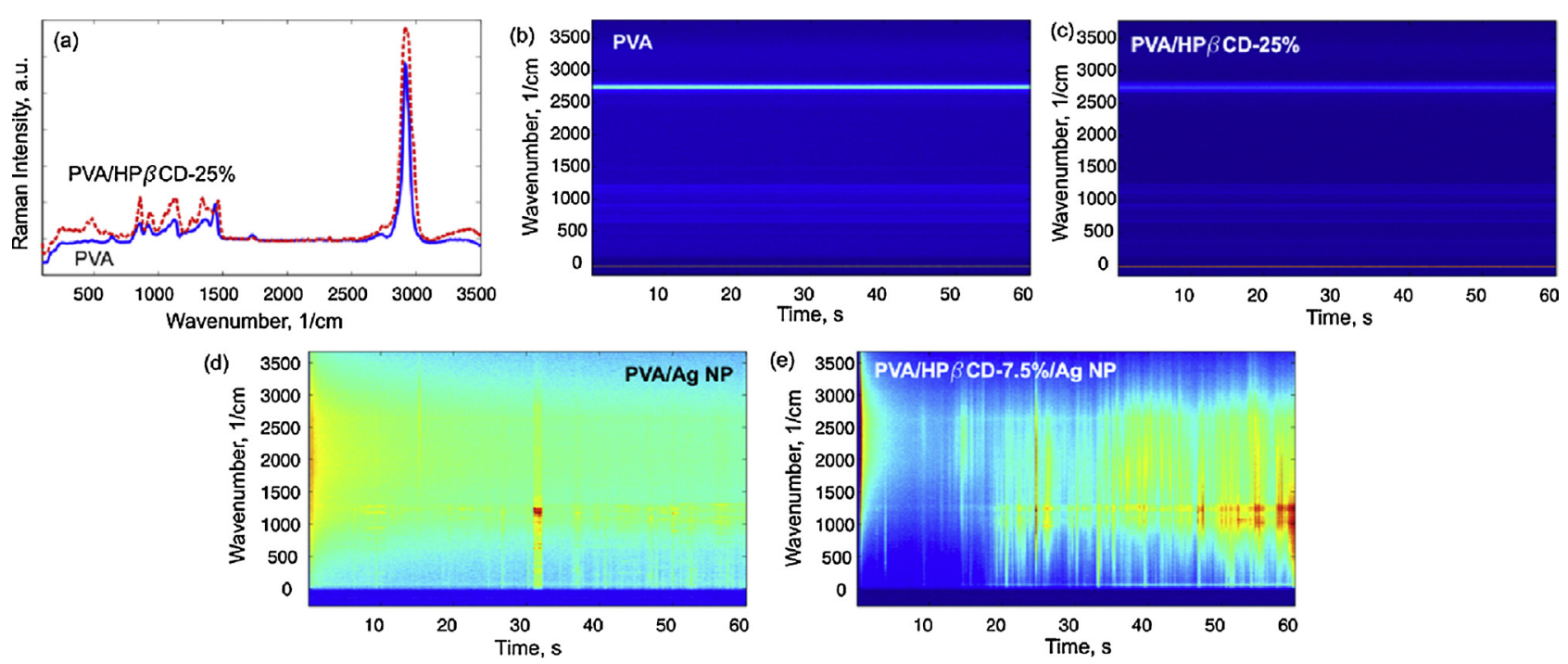

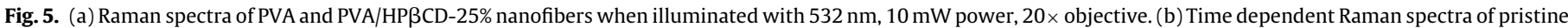

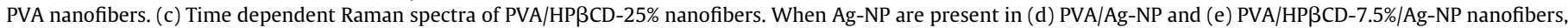

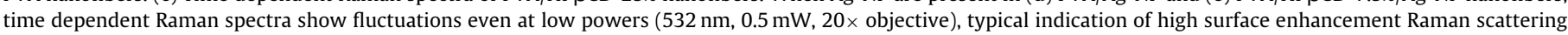
(SERS). Due to high absorption coefficient and high thermal insulation of free standing fibers, absorption causes thermal damage to sample at high powers (e.g. $10 \mathrm{~mW}$ ).

\subsection{Structural characterization of PVA/Ag-NP and $P V A / H P \beta C D / A g-N P$ composite nanofibers}

The characteristics of Ag-NP were investigated by using HRTEM and XRD measurements. The representative HR-TEM, given in Fig. 3a as an inset figure, shows the lattice fringes of Ag-NP in PVA/Ag-NP composite nanofibers and the $d$-spacing was measured to be $0.235 \mathrm{~nm}$ from the lattice fringes that corresponded to the lattice spacing of the (111) planes of the fcc Ag (Zeng et al., 2012). The XRD patterns of the electrospun nanofibrous mats were given in Fig. 4a. The PVA/Ag-NP sample has diffraction peaks at $2 \theta=38.4^{\circ}$, $44.4^{\circ}, 64.6^{\circ}$ and $77.6^{\circ}$ which belong to (111), (200), (220), and (311) crystal planes of Ag, respectively (Li et al., 2007; Wei, Han, Walker, Fuller, \& Grzybowski, 2012). For PVA/HPßCD/Ag-NP samples same characteristic peaks of elemental Ag were observed, but, there were also additional peaks at $2 \theta=28.0^{\circ}, 32.4^{\circ}, 46.4^{\circ}, 55.0^{\circ}$ and $57.9^{\circ}$ which correspond to existing of oxidized $\mathrm{Ag}$ (Chen et al., 2006; Singh, Mehta, Joshi, Kruis, \& Shivaprasad, 2007; Wei et al., 2012). This is possibly originated from the oxidation of unsaturated outer surface of Ag-NP by the hydroxyl groups of HP $\beta C D$ molecules (Porramezan \& Eisazadeh, 2011). For PVA/HPßCD-25\%/Ag-NP, it was also observed that the diffraction peak intensity for $\mathrm{Ag}$ was decreased significantly and $\mathrm{Ag}_{2} \mathrm{O}$ peaks became prominent suggesting that Ag-NP has higher oxized content since this sample contains the highest amount of HP $\beta C D$.

The UV-vis absorption measurements of the PVA/Ag-NP and PVA/HP $\beta C D / A g-N P$ were obtained from the aqueous solution by dissolving the nanofibrous mats in water (Fig. 4b). For all samples, the spectra show an absorption band at the range of $400-450 \mathrm{~nm}$ wavelengths due to the characteristic surface plasmonic resonance (SPR) band for $\mathrm{Ag}-\mathrm{NP}$ (Ng et al., 2008; Wu et al., 2011). For PVA/Ag-NP nanofibers the particle size was $8.0 \pm 0.5 \mathrm{~nm}$ and the maximum absorption value exists at $421 \mathrm{~nm}$. On the other hand, the SPR peak for PVA/HP $\beta C D-7.5 \% / \mathrm{Ag}-\mathrm{NP}$ (APS $=2.7 \pm 0.5 \mathrm{~nm}$ ) was observed at $439 \mathrm{~nm}$. As the HP $\beta C D$ content increased, blue-shift of absorption peaks to $435 \mathrm{~nm}$ and $428 \mathrm{~nm}$ for PVA/HP $\beta C D-15 \% / \mathrm{Ag}-\mathrm{NP}$ (APS $=2.6 \pm 0.5 \mathrm{~nm}$ ) and PVA/HP $\beta C D-$ $25 \% / \mathrm{Ag}-\mathrm{NP}$ (APS $=1.8 \pm 0.4 \mathrm{~nm}$ ) were observed, respectively due to the decreasing size of Ag-NP in the fiber matrix. Even though the Ag-NP particle size is distinctively smaller for the PVA nanofibers containing HP $\beta C D$ compared to HP $\beta C D$ free PVA nanofibers, the absorption peak of the spectrum firstly shows red-shift to the higher wavelength and broadening for PVA/HP $\beta C D / A g-N P$ systems. This can be explained by the existence $\mathrm{Ag}_{2} \mathrm{O}$ on the $\mathrm{Ag}-\mathrm{NP}$ that was also proved by the XRD measurements. For the PVA/HP $\beta C D / A g-$ $\mathrm{NP}$ samples, the $\mathrm{Ag}_{2} \mathrm{O}$ layer on the $\mathrm{Ag}-\mathrm{NP}$ surface results an absorption shoulder at $450-500 \mathrm{~nm}$ range and it also causes broadening at the characteristic absorption band of Ag-NP (Chen et al., 2006). Fig. 4c shows the XPS spectra of PVA/Ag-NP and PVA/HP $\beta C D / A g-N P$ nanofibers. The doublet peaks at 368.2 and $374.2 \mathrm{eV}$ are assigned to the binding energies $3 \mathrm{~d}_{5 / 2}$ and $3 \mathrm{~d}_{3 / 2}$ of $\mathrm{Ag}$ 3d core level, respectively (Suh, Moon, Lee, \& Jang, 2006). There are merely differences between the binding energy of $\mathrm{Ag}^{0}$ and $\mathrm{Ag}^{1+}$, so the ionic type existence cannot be easily noticed from $\mathrm{Ag} 3 \mathrm{~d}$ core level which was supported by XRD measurements (Li et al., 2007).

\subsection{Surface enhanced Raman scattering (SERS) properties of PVA/Ag-NP and PVA/HP $\beta C D / A g-N P$ composite nanofibers}

Surface enhanced Raman scattering (SERS) properties of Ag-NP surface-decorated electrospun polymeric nanofibers has been previously demonstrated, resulting in flexible SERS active substrates (Zhang et al., 2012). We investigated the presence of SERS effects in Ag-NP loaded PVA and PVA/HP $\beta C D$ nanofibers as shown in Fig. 5. Without Ag-NP, the PVA and PVA/HP $\beta C D$ nanofiber samples exhibited Raman spectra (Fig. 5a), that are stable in time, even under high excitation powers of $10 \mathrm{~mW}$ (Fig. $5 \mathrm{~b}$ and c). However, PVA/Ag-NP (Fig. 5d) and PVA/HPßCD/Ag-NP (Fig. 5e) nanofibers exhibited fluctuating Raman spectra even at low powers ( $<1 \mathrm{~mW}$ ) due to the presence of Ag-NP, typical indication of strong SERS effect. Time dependent Raman spectra of PVA/HP $\beta C D-7.5 \% / A g-N P$ sample was given as example in Fig. 5e, but, PVA/HP $3 C D-15 \% / A g-$ NP and PVA/HP $\beta C D-25 \% / A g-N P$ samples have also shown similar characteristics (data not shown). It has been also observed that (data not shown), high excitation powers resulted in thermal damage to PVA/Ag-NP and PVA/HP $\beta C D / A g-N P$ nanofibrous mats due to poor thermal conduction of nanofibers and high absorption of Ag-NP. Fluctuations of the SERS signal from Ag-NP incorporated nanofibers suggest that a thermally activated mechanism is likely to be the source of observed blinking behavior. In short, PVA/Ag-NP 


\section{(a)}

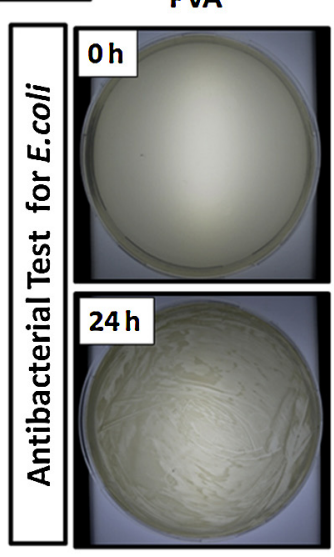

PVA/HPßCD-25\%

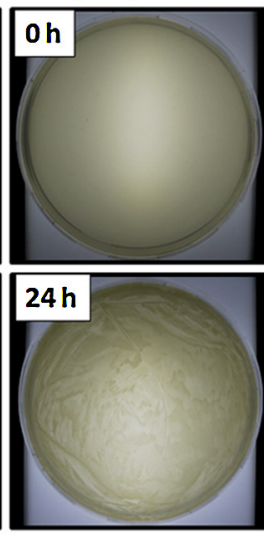

\section{(b)}

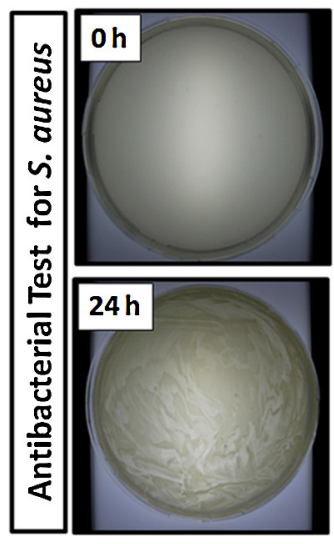

PVA/HPßCD-25\%

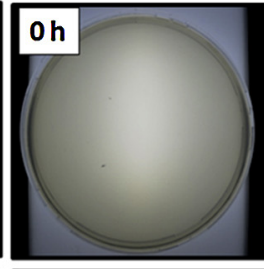

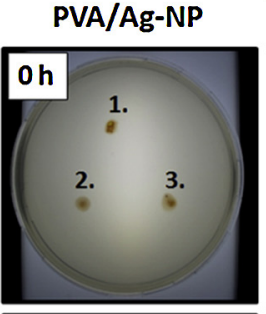

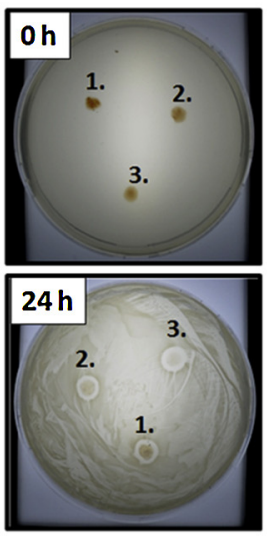

PVA/HPßCD-7.5\%/ PVA/HPßCD-15\%/

PVA/HPßCD-25\%/

PVA/HPBCD-7.
Ag-NP

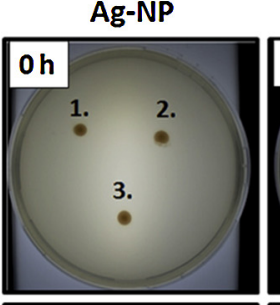

Ag-NP
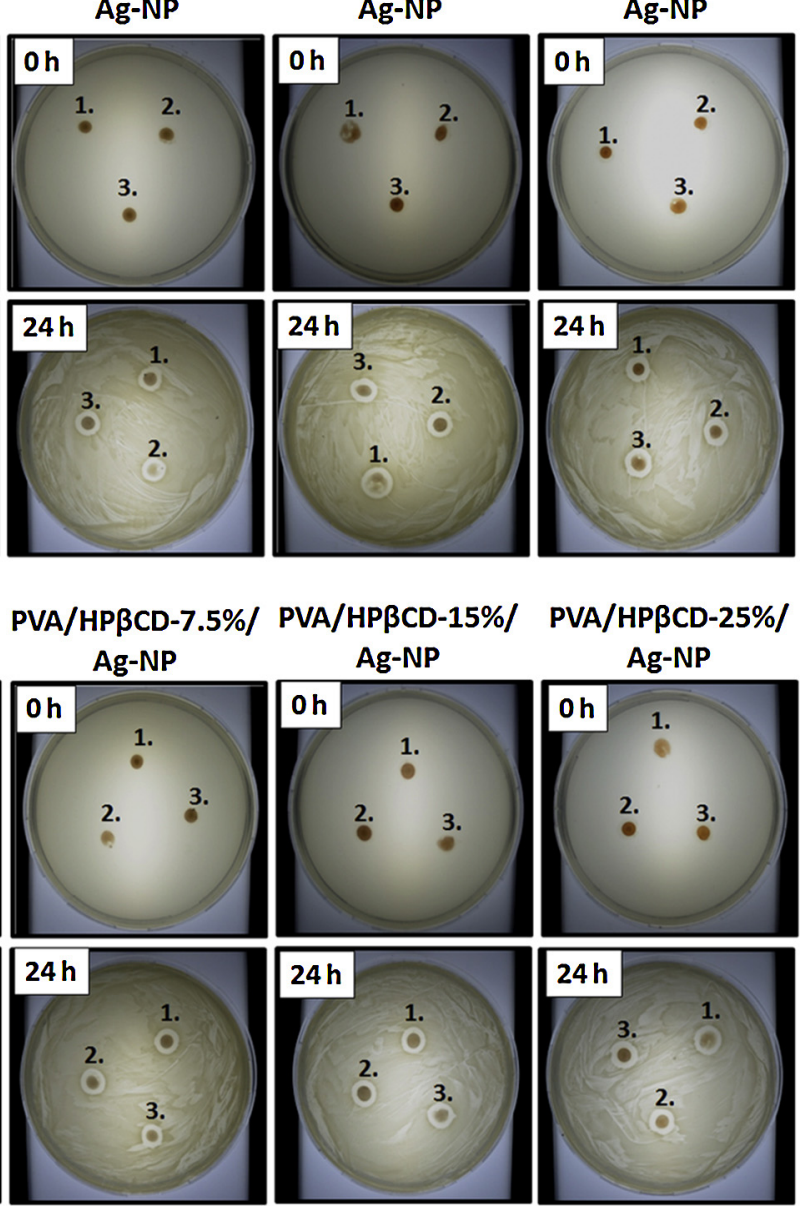

PVA/HPBCD-15\%/

PVA/HPßCD-25\%/

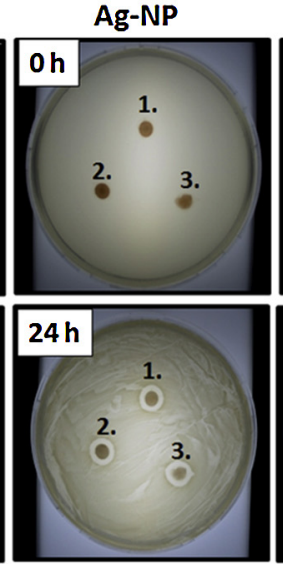

Ag-NP
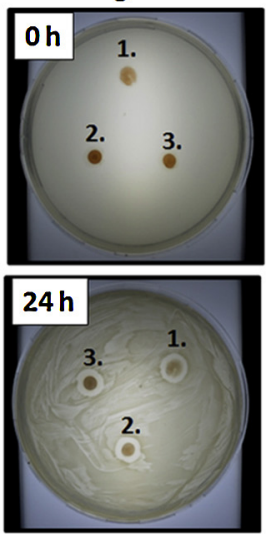

Fig. 6. The photographs of antibacterial testing of nanofibrous mats which were performed against (a) E. coli and (b) S. aureus.

and PVA/HP $\beta C D / A g-N P$ samples exhibited SERS effect which can be useful for sensing application (Zhang et al., 2012).

\subsection{Antibacterial properties of PVA/Ag-NP and $P V A / H P \beta C D / A g-N P$ composite nanofibers}

Here, we investigated the antibacterial effects of PVA/Ag-NP and PVA/HP $\beta C D / A g-N P$ nanofibrous mats against Gram-negative (E. coli) and Gram-positive (S. aureus) bacteria. The electrospun PVA and PVA/HP $\beta C D$ without Ag-NP were also tested for comparison. For the antibacterial test, three samples taken from different locations of the same nanofibrous mat were placed on $E$. coli and $S$. aureus spreaded agar plates and visualized after incubation for $24 \mathrm{~h}$. The plates were checked for the presence and size of the inhibition zones (Fig. 6 and Table 2). We observed that,
PVA and PVA/HPßCD nanofibers did not show any antibacterial activity. On the other hand, PVA/Ag-NP nanofibers have shown antibacterial property due to the presence of Ag-NP. Ag-NP has antibacterial property, which is size dependent (Morones et al., 2005). So, in the case of PVA/HP $\beta C D / A g-N P$ nanofibers, as the size of Ag-NP decreased depending on the HP $\beta C D$ amount, the inhibition zone became larger indicating enhanced antibacterial activity compared to PVA/Ag-NP nanofibers (Fig. 6). Among PVA/HPßCD/Ag-NP samples, the PVA/HP $\beta C D-25 \% / A g-N P$ has shown better antibacterial activity since the Ag-NP has the smallest size in this sample (Table 1). This may be due to the fact that the release of the $\mathrm{Ag}$-NP becomes easier as the particle size decreases, so that AgNP can more effectively reach the bacteria region and contact with the bacteria. In addition, owing to the smaller dimensions, higher surface to volume ratios were obtained which also enhances

Table 2

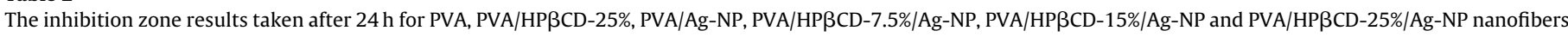
against E. coli and S. aureus.

\begin{tabular}{|c|c|c|c|c|}
\hline \multirow[t]{2}{*}{ Samples } & \multicolumn{2}{|l|}{ E. coli } & \multicolumn{2}{|l|}{ S. aureus } \\
\hline & $0 \mathrm{~h}(\mathrm{~cm})$ & $24 \mathrm{~h}(\mathrm{~cm})$ & $0 \mathrm{~h}(\mathrm{~cm})$ & $24 \mathrm{~h}(\mathrm{~cm})$ \\
\hline PVA & 0.8 & 0.8 & 0.8 & 0.8 \\
\hline PVA/HP $\beta C D-25 \%$ & 0.8 & 0.8 & 0.8 & 0.8 \\
\hline PVA/Ag-NP & 0.8 & $1.32 \pm 0.00$ & 0.8 & $1.41 \pm 0.15$ \\
\hline PVA/HP $\beta C D-7.5 \% / A g-N P$ & 0.8 & $1.55 \pm 0.07$ & 0.8 & $1.62 \pm 0.16$ \\
\hline PVA/HP $\beta C D-15 \% / A g-N P$ & 0.8 & $1.60 \pm 0.03$ & 0.8 & $1.73 \pm 0.21$ \\
\hline PVA/HP $\beta C D-25 \% / A g-N P$ & 0.8 & $1.72 \pm 0.16$ & 0.8 & $1.85 \pm 0.07$ \\
\hline
\end{tabular}


the antibacterial activity of Ag-NP (Martinez-Castanon, NinoMartinez, Martinez-Gutierrez, Martinez-Mendoza, \& Ruiz, 2008; Mollahosseini, Rahimpour, Jahamshahi, Peyravi, \& Khavarpour, 2012). Similarly, Morones et al. reported that bactericidal properties of the Ag-NP are size dependent and smaller Ag-NP were much more effective against Gram-negative bacteria since Ag-NP having a diameter of $\sim 1-10 \mathrm{~nm}$ preferentially present a direct interaction with the bacteria (Morones et al., 2005). Moreover, we observed that the increase in the inhibition zone was more distinctive for $S$. aureus compared to E. coli as the Ag-NP size get smaller and this could be attributed to the cellular wall content differences between Gram-positive and Gram-negative bacteria as discussed by Thiel et al. (2007).

\section{Conclusion}

Here, we have achieved one-step synthesis of Ag-NP by reduction of $\mathrm{AgNO}_{3}$ by using PVA and PVA/HPßCD aqueous solution as reducing and stabilizing medium as well as the electrospinning matrix. For the PVA/Ag-NP system, Ag-NP with an APS of $8.0 \pm 0.5 \mathrm{~nm}$ was obtained and a small number of Ag-NP aggregation was observed within the PVA fiber matrix. The size of Ag-NP decreased significantly with the addition of HP $\beta C D$ to the PVA solution and homogeneous distribution of Ag-NP without aggregation was achieved for the PVA/HP $\beta C D / A g-N P$ nanofibers. By increasing the amount of HP $\beta C D$ in the PVA solution, the size-tunable AgNP synthesis was successful owing to the efficient reducing and stabilizing properties of HP $\beta C D$. The size of the Ag-NP in PVA/AgNP nanofibers was decreased to $2.7 \pm 0.5 \mathrm{~nm}, 2.6 \pm 0.5 \mathrm{~nm}$ and $1.8 \pm 0.4 \mathrm{~nm}$ for PVA/HPßCD-7.5\%/Ag-NP, PVA/HPßCD-15\%/Ag-NP and PVA/HP $\beta C D-25 \% / \mathrm{Ag}-\mathrm{NP}$ nanofibers, respectively. We have observed that, multifunctional PVA/Ag-NP and PVA/HP $\beta C D / A g-$ NP nanofibers exhibited SERS effect which might be applicable in sensing applications, in addition, these nanofibrous mats have shown antibacterial effect against $E$. coli and $S$. aureus bacteria. When compared to PVA/Ag-NP, PVA/HPßCD/Ag-NP samples have shown better antibacterial efficiency due to smaller size of Ag-NP. In addition, the PVA/HP $\beta C D-25 \% / \mathrm{Ag}-\mathrm{NP}$ having the smaller Ag-NP has shown better antibacterial efficiency among PVA/HP $\beta C D / A g-$ NP samples. In brief, our approach is a "green" and facile method for the fabrication of Ag-NP incorporated functional nanofibrous mats having SERS effect and antibacterial properties. HP $\beta C D$ is a bio-compatible and non-toxic oligosaccharide and the additional use of hazardous reducing/stabilizing agent for the formation of Ag-NP can be eliminated by using HP $\beta C D$. Furthermore, polymer matrix such as PVA is also known for its biocompatible nature and suitable for biomedical applications. Therefore, these electrospun PVA/HP $\beta C D / A g-N P$ nanofibrous mats can be quite applicable as a wound healing material, in sensing or other biomedical uses.

\section{Acknowledgements}

State Planning Organization (DPT) of Turkey is acknowledged for the support of UNAM-Institute of Materials Science \& Nanotechnology. Dr T. Uyar acknowledges EU FP7-Marie Curie-IRG for funding NANOWEB (PIRG06-GA-2009-256428). A. Celebioglu acknowledges TUBITAK-BIDEB for National PhD Scholarship.

\section{References}

Alvarez, J., Liu, J., Román, E., \& Kaifer, A. E. (2000). Water-soluble platinum and palladium nanoparticles modified with thiolated $\beta$-cyclodextrin. Chemical Communication, 13, 1151-1152.

Arvizo, R. R., Bhattacharyya, S., Kudgus, R. A., Giri, K., Bhattacharya, R., \& Mukherjee, P. (2012). Intrinsic therapeutic applications of noble metal nanoparticles: Past, present and future. Chemical Society Reviews, 41(7), 2943-2970.
Cai, J., Kimura, S., Wada, M., \& Kuga, S. (2009). Nanoporous cellulose as metal nanoparticles support. Biomacromolecules, 10(1), 87-94.

Celebioglu, A., \& Uyar, T. (2012). Electrospinning of nanofibers from non-polymeric systems: Polymer-free nanofibers from cyclodextrin derivatives. Nanoscale, 4(2), 621-631.

Chae, H. H., Kim, B.-H., Yang, K. S., \& Rhee, J. I. (2011). Synthesis and antibacterial performance of size-tunable silver nanoparticles with electrospun nanofiber composites. Synthetic Metals, 161(19), 2124-2128.

Chen, M., Wang, L.-Y., Han, J.-T., Zhang, J.-Y., Li, Z.-Y., \& Qian, D.-J. (2006). Preparation and study of polyacryamide-stabilized silver nanoparticles through a one-pot process. Journal of Physical Chemistry B, 110(23), 11224-11231.

Chung, J. W., Guo, Y., Priestley, R. D., \& Kwak, S.-Y.(2011). Colloidal gold nanoparticle formation derived from self-assembled supramolecular structure of cyclodextrin/Au salt complex. Nanoscale, 3(4), 1766-1772.

Fouda, M. M. G., El-Aassar, M. R., \& Al-Deyab, S. S. (2013). Antimicrobial activity of carboxymethyl chitosan/polyethylene oxide nanofibers embedded silver nanoparticles. Carbohydrate Polymers, 92(2), 1012-1017.

Greiner, A., \& Wendorff, J. H. (2007). Electrospinning: A fascinating method for the preparation of ultrathin fibers. Angewandte Chemie International Edition, 46(30), 5670-5703.

Guiping, M., Dawei, F., Yang, L., Xiaodan, Z., \& Jun, N. (2012). Electrospun sodium alginate/poly(ethylene oxide) core-shell nanofibers scaffolds potential for tissue engineering applications. Carbohydrate Polymers, 87(1), 737-743.

Guzman, M., Dille, J., \& Godet, S. (2012). Synthesis and antibacterial activity of silver nanoparticles against gram-positive and gram-negative bacteria. Nanomedicine: Nanotechnology, Biology and Medicine, 8(1), 37-45.

Hang, A. T., Tae, B., \& Park, J. S. (2010). Non-woven mats of poly(vinyl alcohol)/chitosan blends containing silver nanoparticles: Fabrication and characterization. Carbohydrate Polymers, 82(2), 472-479.

Harada, A., Kobayashi, R., Takashima, Y., Hashidzume, A., \& Yamaguchi, H. (2010). Macroscopic self-assembly through molecular recognition. Nature Chemistry, 3(1), 34-37.

Hedges, A. R. (1998). Industrial applications of cyclodextrins. Chemical Reviews, 98(5), 2035-2044

Huang, T., Meng, F., \& Qi, L. (2009). Facile synthesis and one-dimensional assembly of cyclodextrin-capped gold nanoparticles and their applications in catalysis and surface-enhanced Raman scattering. Journal of Physical Chemistry C, 113(31), $13636-13642$

Jin, M., Zhang, X., Nishimoto, S., Liu, Z., Tryk, D. A., Murakami, T., et al. (2007) Large-scale fabrication of Ag nanoparticles in PVP nanofibres and net-like silver nanofibre films by electrospinning. Nanotechnology, 18(7), 075605.

Kochkar, H., Aouine, M., Ghorbel, A., \& Berhault, G. (2011). Shape-controlled synthesis of silver and palladium nanoparticles using $\beta$-cyclodextrin. Journal of Physical Chemistry C, 115(23), 11364-11373.

Laudenslager, M. J., Schiffman, J. D., \& Schauer, C. L. (2008). Carboxymethyl chitosan as a matrix material for platinum, gold, and silver nanoparticles. Biomacromolecules, 9(10), 2682-2685.

Li, D., \& Xia, Y. (2004). Electrospinning of nanofibers: Reinventing the wheel? Advanced Materials, 16(14), 1151-1170.

Li, S., Shen, Y., Xie, A., Yu, X., Qiu, L., Zhang, L., et al. (2007). Green synthesis of silver nanoparticles using Capsicum annuum L. extract. Green Chemistry, 9(8), $852-858$.

Li, Z., Huang, H., Shang, T., Yang, F., Zheng, W., Wang, C., et al. (2006). Facile synthesis of single-crystal and controllable sized silver nanoparticles on the surfaces of polyacrylonitrile nanofibres. Nanotechnology, 17(3), 917-920.

Liu, W., Herrmann, A. K., Geiger, D., Borchardt, L., Simon, F., Kaskel, S., et al. (2012) High performance electrocatalysis on palladium aerogels. Angewandte Chemie International Edition, 51(23), 5743-5747.

Mahanta, N., \& Valiyaveettil, S. (2012). In situ preparation of silver nanoparticles on biocompatible methacrylated poly(vinyl alcohol) and cellulose based polymeric nanofibers. RSC Advances, 2(30), 11389-11396.

Martinez-Castanon, G., Nino-Martinez, N., Martinez-Gutierrez, F., MartinezMendoza, J., \& Ruiz, F. (2008). Synthesis and antibacterial activity of silver nanoparticles with different sizes. Journal of Nanoparticle Research, 10(8), 1343-1348.

Mollahosseini, A., Rahimpour, A., Jahamshahi, M., Peyravi, M., \& Khavarpour, M. (2012). The effect of silver nanoparticle size on performance and antibacteriality of polysulfone ultrafiltration membrane. Desalination, 306, 41-50.

Morones, J. R., Elechiguerra, J. L., Camacho, A., Holt, K., Kouri, J. B., Ramírez, J. T., et al. (2005). The bactericidal effect of silver nanoparticles. Nanotechnology, 16(10), 2346.

Nam, S. H., Shim, H.-S., Kim, Y.-S., Dar, M. A., Kim, J. G., \& Kim, W. B. (2010). Ag or Au nanoparticle-embedded one-dimensional composite $\mathrm{TiO}_{2}$ nanofibers prepared via electrospinning for use in lithium-ion batteries. ACS Applied Materials E'Interfaces, 2(7), 2046-2052.

Ng, C. H. B., Yang, J., \& Fan, W. Y. (2008). Synthesis and self-assembly of onedimensional sub-10 nm Ag nanoparticles with cyclodextrin. Journal of Physical Chemistry C, 112(11), 4141-4145.

Patel, A. C., Li, S., Wang, C., Zhang, W., \& Wei, Y. (2007). Electrospinning of porous silica nanofibers containing silver nanoparticles for catalytic applications. Chemistry of Materials, 19(6), 1231-1238.

Porramezan, M., \& Eisazadeh, H. (2011). Fabrication and characterization of polyaniline nanocomposite modified with $\mathrm{Ag}_{2} \mathrm{O}$ nanoparticles. Composites Part B: Engineering, 42(7), 1980-1986.

Rai, M., Yadav, A., \& Gade, A. (2009). Silver nanoparticles as a new generation of antimicrobials. Biotechnology Advances, 27(1), 76-83. 
816

A. Celebioglu et al. / Carbohydrate Polymers 99 (2014) 808-816

Ramakrishna, S. (2005). An introduction to electrospinning and nanofibers. World Scientific Publishing Company.

Ramakrishna, S., Fujihara, K., Teo, W.-E., Yong, T., Ma, Z., \& Ramaseshan, R. (2006) Electrospun nanofibers: Solving global issues. Materials Today, 9(3), 40-50.

Ravi, S. S., Christena, L. R., SaiSubramanian, N., \& Anthony, S. P. (2013). Green synthesized silver nanoparticles for selective colorimetric sensing of $\mathrm{Hg}^{2+}$ in aqueous solution at wide pH range. Analyst, 138(15), 4370-4377.

Rycenga, M., Cobley, C. M., Zeng, J., Li, W., Moran, C. H., Zhang, Q., et al. (2011). Controlling the synthesis and assembly of silver nanostructures for plasmonic applications. Chemical Reviews, 111(6), 3669.

Saquing, C. D., Manasco, J. L., \& Khan, S. A. (2009). Electrospun nanoparticle-nanofiber composites via a one-step synthesis. Small, 5(8), 944-951.

Shervani, Z., \& Yamamoto, Y. (2011). Carbohydrate-directed synthesis of silver and gold nanoparticles: Effect of the structure of carbohydrates and reducing agents on the size and morphology of the composites. Carbohydrate Research, 346(5), 651-658.

Shi, Q., Vitchuli, N., Nowak, J., Noar, J., Caldwell, J. M., Breidt, F., et al. (2011). One-step synthesis of silver nanoparticle-filled nylon 6 nanofibers and their antibacterial properties. Journal of Materials Chemistry, 21(28), 10330-10335.

Singh, V. N., Mehta, B. R., Joshi, R. K., Kruis, F. E., \& Shivaprasad, S. M. (2007). Enhanced gas sensing properties of $\mathrm{In}_{2} \mathrm{O}_{3}: \mathrm{Ag}$ composite nanoparticle layers; Electronic interaction, size and surface induced effects. Sensors and Actuators B: Chemical, 125(2), 482-488.

Suh, M. P., Moon, H. R., Lee, E. Y., \& Jang, S. Y. (2006). A redox-active two-dimensional coordination polymer: Preparation of silver and gold nanoparticles and crystal dynamics on guest removal. Journal of the American Chemical Society, 128(14), 4710-4718.

Szejtli,J. (1998). Introduction and general overview of cyclodextrin chemistry. Chemical Reviews, 98(5), 1743.

Thiel, J., Pakstis, L., Buzby, S., Raffi, M., Ni, C., Pochan, D. e. J., et al. (2007). Antibacterial properties of silver-doped titania. Small, 3(5), 799-803.
Wang, S., Bai, J., Li, C., \& Zhang, J. (2012). Functionalization of electrospun $\beta$ cyclodextrin/polyacrylonitrile (PAN) with silver nanoparticles: Broad-spectrum antibacterial property. Applied Surface Science, 261, 499-503.

Wang, S., Bai, J., Li, C., Zhang, Y., \& Zhang, J. (2012). Ag nanoparticle-embedded one-dimensional $\beta$-CD/PVP composite nanofibers prepared via electrospinning for use in antibacterial material. Colloid and Polymer Science, 290(7), 667-672.

Wei, Y., Han, S., Walker, D. A., Fuller, P. E., \& Grzybowski, B. A. (2012). Nanoparticle core/shell architectures within MOF crystals synthesized by reaction diffusion. Angewandte Chemie International Edition, 51(30), 7435-7439.

Wendorff, J. H., Agarwal, S., \& Greiner, A. (2012). Electrospinning: Materials, processing, and applications. Wiley-VCH.

Wu, H., He, L., Gao, M., Gao, S., Liao, X., \& Shi, B. (2011). One-step in situ assembly of size-controlled silver nanoparticles on polyphenol-grafted collagen fiber with enhanced antibacterial properties. New Journal of Chemistry, 35(12), 2902-2909.

Xiao, S., Xu, W., Ma, H., \& Fang, X. (2012). Size-tunable Ag nanoparticles immobilized in electrospun nanofibers: Synthesis, characterization, and application for catalytic reduction of 4-nitrophenol. RSC Advances, 2(1), 319-327.

Xu, X., Yang, Q., Wang, Y., Yu, H., Chen, X., \& Jing, X. (2006). Biodegradable electrospun poly(L-lactide) fibers containing antibacterial silver nanoparticles. European Polymer Journal, 42(9), 2081-2087.

Zeng, J., Zhu, C., Tao, J., Jin, M., Zhang, H., Li, Z. Y., et al. (2012). Controlling the nucleation and growth of silver on palladium nanocubes by manipulating the reaction kinetics. Angewandte Chemie International Edition, 51(10), 2354-2358.

Zhang, L., Gong, X., Bao, Y., Zhao, Y., Xi, M., Jiang, C., et al. (2012). Electrospun nanofibrous membranes surface-decorated with silver nanoparticles as flexible and active/sensitive substrates for surface-enhanced Raman scattering. Langmuir, 28(40), 14433-14440.

Zhu, H., Du, M., Zou, M., Xu, C., Li, N., \& Fu, Y. (2012). Facile and green synthesis of well-dispersed Au nanoparticles in PAN nanofibers by tea polyphenols. Journal of Materials Chemistry, 22(18), 9301-9307. 